\section{Chloroquine Toxicity Misdiagnosed as Fabry Disease Associated with Systemic Lupus Erythematosus and Hashimoto Thyroiditis}

\section{To the Editor:}

Fabry disease or Anderson-Fabry disease is an X-linked recessive lysosome storage disease resulting from the deficiency of the $\alpha$-galactosidase enzyme $(\alpha-G a l A)^{1}$. Low activity of the enzyme leads to abnormal lysosomal accumulation of globotriaosylceramide in many organs such as kidneys, and arterial walls, cardiac muscles, and nerve cells. While men with $<1 \%$ $\alpha$-Gal A enzyme typically experience symptoms of acroparesthesias, and the appearance of angiokeratomas, hypohidrosis, characteristic corneal and lenticular opacities (cornea verticillata), and proteinuria, women (homozygous and heterozygous) can range from being asymptomatic to having severe symptoms ${ }^{1}$. Literature reports a coexistence of Fabry disease with autoimmune diseases such as systemic lupus erythematosus (SLE), rheumatoid arthritis, and $\operatorname{IgA}$ nephropathy ${ }^{2}$.

Case presentation. A 14-year-old girl was diagnosed with SLE and Hashimoto thyroiditis 1.5 years ago, based on the history of recurrent subfebrile episodes, arthralgia, fatigue, pain and numbness in hands, and hair effluvium. Laboratory investigations revealed anemia, thrombocytopenia $\left(122 \times 10^{9} / \mathrm{l}\right)$, hematuria $\left(133 \mathrm{E} / \mathrm{mm}^{3}\right)$, and proteinuria $(190 \mathrm{mg} /$ day $)$, while immunological findings confirmed hypocomplementemia (C3 0.79 $\mathrm{g} / \mathrm{l}$ ), positive anticardiolipin antibody ( $\mathrm{aCL}$; IgG 23.9 phospholipid units $/ \mathrm{ml}$, aCL IgM 56.1 phospholipid units/ml), antinuclear antibodies (1:1280), and anti-dsDNA antibodies (50.8 IU/ml). During the followup, she was referred to a rheumatological and nephrological reevaluation because of the progression of lumbar pain and proteinuria. She further complained of vertigo, tinnitus, chronic diarrhea, and pain in the extremities. The patient has been treated with prednisolone ( $10 \mathrm{mg}$ daily), azathioprine $(2 \times 50 \mathrm{mg}$ daily), and hydroxychloroquine (HCQ; $500 \mathrm{mg}$ daily) for SLE, and with levothyroxine for Hashimoto thyroiditis. On presentation, the patient was subfebrile with a cheekbone rash, proximal muscle weakness and atrophy, bilateral knee and ankle edema, and a palpable liver. Family history has revealed that her mother has also had recurrent episodes of fever, diarrhea, vertigo, arthralgia, and pain and numbness in hands since childhood. Her mother's physical examination revealed skin angiokeratomas in the gluteal region and lower abdomen. Both of her sisters have autoimmune thrombocytopenia. At that time, the patient had proteinuria up to $1 \mathrm{~g} /$ day with glomerular filtration rate at $151.8 \mathrm{ml} / \mathrm{min}$.

A renal biopsy was performed and immunofluorescence studies demonstrated minor granular deposition of IgM limited to mesangial areas and granular deposition $\mathrm{C} 3$ in tunica intima of blood vessels. Electronic microscope studies showed numerous zebra structures, mainly located in the cytoplasm of podocytes in association with mesangial dense deposits. The presence of zebra bodies led to the initial suspicion of Fabry disease, but subsequent enzyme assays revealed normal leukocyte $\alpha$-Gal A activity, and genetic analysis did not demonstrate GLA gene mutation, ruling out the diagnosis. However, further analysis has revealed curvilinear bodies in renal cells, supporting the diagnosis of HCQ-induced renal phospholipidosis. On that basis, HCQ has been discontinued and the followup, 6 months later, has shown the patient's proteinuria resolved and her muscle function improved. A control renal biopsy was suggested, but not accepted by the patient's mother.

Presented here is the case of a female adolescent with Hashimoto thyroiditis and SLE with renal impairment and proteinuria, whereby a renal biopsy led to an unexpected and instructive diagnosis.
To our knowledge, few cases of the coexistence of Fabry disease and SLE have been reported in the literature $3,4,5$. Likewise, there are only a few cases of chloroquine toxicity misdiagnosed as Fabry disease in patients with different rheumatic diseases ${ }^{6,7,8,9}$. However, tissue damage induced by chloroquine has many morphological similarities to Fabry disease, leading to misinterpretations of pathological findings in the case of an ambiguous medical history, or in a situation in which all relevant data regarding medical therapy are not available to the interpreting pathologist ${ }^{8,10}$.

The coexistence of Fabry disease, SLE, and Hashimoto thyroiditis is extremely rare. As a result of an overenthusiastic chase for the infrequent disease, we were mistaken in our diagnostic reasoning and failed to take into account all other possible causes of renal damage.

MARTA NAVRATIL, MD, PhD; IRENA IVKOVIĆ JUREKOVIĆ, MD, PhD, Department of Pulmonology, Allergology, Rheumatology and Clinical Immunology, Children's Hospital Zagreb, Zagreb, and Faculty of Medicine, University of Osijek, Osijek, Croatia. Address correspondence to Dr. M. Navratil, Department of Pulmonology, Allergology, Rheumatology and Clinical Immunology, Children's Hospital Zagreb, Klaićeva 16, 10000 Zagreb, Croatia. E-mail: marta.navratil@gmail.com

\section{REFERENCES}

1. Mehta A, Hughes DA. Fabry disease. In: Pagon RA, Bird TC, Dolan CR, Stephens K, editors. GeneReviews. Updated Jan 5, 2017. [Internet. Accessed September 21, 2017.] Available from: www.ncbi.nlm.nih.gov/books/NBK1292/

2. Martinez P, Aggio M, Rozenfeld P. High incidence of autoantibodies in Fabry disease patients. J Inherit Metab Dis 2007;30:365-9.

3. Majima K, Ishizaki T, Inoue T, Hori Y, Egami J, Oohara A, et al. A case of Fabry's disease associated with lupus nephritis. Nippon Jinzo Gakkai Shi 1992;34:1189-94.

4. Rahman P, Gladman DD, Wither J, Silver MD. Coexistence of Fabry's disease and systemic lupus erythematosus. Clin Exp Rheumatol 1998;16:475-8.

5. Waggoner T, Waggoner K, Guma M, Un Kim D, Byrd L. Clinical mimicry: Fabry disease masquerading as lupus? [Internet. Accessed September 21, 2017.] Available from: www.physicianspractice.com/ lupus/clinical-mimicry-fabry-disease-masquerading-lupus

6. Albay D, Adler SG, Philipose J, Calescibetta CC, Romansky SG, Cohen AH. Chloroquine-induced lipidosis mimicking Fabry disease. Mod Pathol 2005;18:733-8.

7. Linthorst GE, Hollak CE. Chloroquine-induced phospholipidosis of the kidney mimicking Fabry's disease. Hum Pathol 2003;34:1358.

8. Müller-Höcker J, Schmid H, Weiss M, Dendorfer U, Braun GS. Chloroquine-induced phospholipidosis of the kidney mimicking Fabry's disease: case report and review of the literature. Hum Pathol 2003;34:285-9.

9. Costa RM, Martul EV, Reboredo JM, Cigarrán S. Curvilinear bodies in hydroxychloroquine-induced renal phospholipidosis resembling Fabry disease. Clin Kidney J 2013;6:533-6.

10. Woywodt A, Hellweg S, Schwarz A, Schaefer RM, Mengel M. A wild zebra chase. Nephrol Dial Transplant 2007;22:3074-7.

J Rheumatol 2017;44:12; doi:10.3899/jrheum.170689 